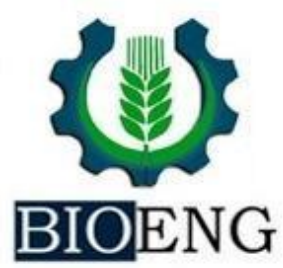

\title{
NÍVEL DE ACEITAÇÃO DA TECNOLOGIA DA INFORMAÇÃO POR PRODUTORES RURAIS
}

\author{
A.C. Cunha ${ }^{1}$, F. F. Putti2 ${ }^{*}$
}

${ }^{1}$ Universidade José Do Rosário Vellano/UNIFENAS - Campus de Alfenas

${ }^{2}$ Universidade Estadual Paulista (UNESP), Faculdade de Ciências e Engenharia - FCE, Campus de Tupã

Article history: Received 24 April 2020; Received in revised form 28 May 2020; Accepted 28 May 2020; Available online 30 September 2020.

\section{RESUMO}

A Tecnologia da Informação tem desempenhado um papel fundamental nos mais diversos setores profissionais, inclusive nos setores agrícolas, trazendo facilidades nos processos, armazenamento de dados, relatórios dos mais diversos tipos e, também, por meio da Internet, consultas e aquisição de conhecimentos. Todavia, nem sempre é realizada a adoção da Tecnologia da Informação por produtores rurais, mesmo tendo em mente as vantagens que ela proporciona. Diante deste contexto, objetivou-se com o presente artigo, analisar o nível de aceitação da tecnologia da informação pelos produtores rurais de algumas cidades da região Sul de Minas Gerais. Para tanto, realizou-se um levantamento de dados por meio de um questionário com 18 questões que foi aplicado com 44 produtores rurais. Por meio dos questionários aplicados, foi possível constatar que grande parte dos agricultores têm acesso à Internet e dispositivos como, por exemplo, computadores. Porém, ainda possuem certa resistência no que tange a adoção da Tecnologia da Informação, mesmo tendo em mente que tal tecnologia poderia auxiliá-los em seus processos de produção.

Palavra-chave: agricultura, computação, internet, software, tecnologia.

\section{LEVEL OF ACCEPTANCE OF INFORMATION TECHNOLOGY BY RURAL PRODUCERS}

\begin{abstract}
Information Technology has played a fundamental role in the most diverse professional sectors, including in the agricultural sectors, providing facilities in the processes, data storage, reports of various types and also through the Internet, consultations and acquisition of knowledge. However, the adoption of Information Technology by rural producers is not always done, even with the advantages it provides. In this context, the objective of this article was to analyze the level of acceptance of information technology by rural producers in some cities in the southern region of Minas Gerais. For that, a data survey was carried out through a questionnaire with 18 questions that was applied with 44 rural producers. Through the questionnaires applied, it was possible to verify that many farmers have access to the Internet and devices such as computers. However, they still
\end{abstract}

*fernando.putti@unesp.br 
have some resistance regarding the adoption of Information Technology, even though it should be borne in mind that such technology could assist them in their production processes.

Index Terms: agriculture, computing, internet, software, technology.

\section{INTRODUÇÃO}

As pessoas, bem como profissionais, vêm buscando cada vez mais simplificar suas tarefas, com a finalidade de economizarem tempo e as concluírem com maior agilidade. Para tanto, vem sendo desenvolvidas novas tecnologias que causam grande impacto em todos os sentidos, inclusive no cenário da agricultura (CUNHA, 2017).

O setor agrícola desempenha um trabalho fundamental no desenvolvimento brasileiro. Há décadas, no Brasil, a agropecuária vem exercendo papeis estratégicos, como aquecimento econômico, geração de empregos e segurança alimentar. (GARCIA; VIEIRA FILHO, 2014). Nos últimos 30 anos, o mundo rural sofreu grandes transformações, fazendo com que haja adaptações por parte dos produtores a este novo cenário. Onde, a produção de sustento passou a ser um sistema agroindustrial. Diante disto, o conhecimento passou a ser um fator de desenvolvimento agrícola e não apenas um privilégio (VIERO; SILVEIRA, 2011).

No ano de 2019, o agronegócio e a agricultura foram responsáveis por $21,4 \%$ do Produto Interno Bruto (PIB) brasileiro, trazendo inúmeras contribuições para a economia, como redução da inflação e aumento de contratações. (CONFEDERAÇÃO DA AGRICULTURA E PECUÁRIA DO BRASIL, 2020).

Para Viero e Silveira (2011), ocorreram-se intensas transformações no meio rural nas últimas três décadas. $\mathrm{O}$ produtor se deparou com a necessidade de adaptar-se, rapidamente, a uma nova realidade, onde a produção de sustento foi substituída pelo sistema agroindustrial. A partir de então, o conhecimento não foi mais um privilégio e passou a ser um agente de desenvolvimento agrícola.

A Tecnologia da Informação tem grande capacidade de aumentar o rendimento e produtividade de processos e, em relação aos produtos agropecuários, proporciona a possibilidade de expandir a quantidade, a qualidade, bem como a inserção destes produtos no mercado. Todavia, sua adesão tem sido limitada devido à alguns fatores como, por exemplo, situação econômica, infraestrutura, falta de conhecimento para utilização da tecnologia e até mesmo baixa escolaridade de pequenos produtores rurais (BAMBINI et al., 2015).

Diante deste cenário, a difusão da Tecnologia da Informação, principalmente da Internet, tornou-se um bem necessário ao ambiente agrícola, assim como já ocorria ao urbano, visando suprir à demanda por conhecimento e informações atualizadas a todo momento (VIERO; SILVEIRA, 2011).

O governo brasileiro, através do Ministério das Comunicações (BR). Portaria No. 431/2009, de 23 de julho de 2009, vem agenciando o Programa Nacional de Telecomunicações Rurais. Sua finalidade é proporcionar à população que reside em áreas rurais o acesso a serviços, de interesse coletivo, de telefonia e Internet (BAMBINI et al., 2015).

O mercado desenvolvedor de softwares, fornece diversas tecnologias voltadas ao setor agrícola, entretanto um número significativo de produtores ainda 
não adotou a tecnologia e executa suas tarefas e relatórios manualmente. Portanto, é de sua importância realizar um estudo para verificar até que ponto os produtores utilizam a tecnologia e que os impedem de adotarem sistemas informatizados que os auxiliem no

\section{MATERIAIS E MÉTODOS}

Para o desenvolvimento do presente artigo, foi realizada uma pesquisa quantitativa, por meio do estudo exploratório e descritivo. Foram selecionados 44 produtores rurais da controle de seus processos. Diante deste contexto, objetivou-se com o presente artigo, analisar o nível de aceitação da tecnologia da informação e o motivo pela não adoção da tecnologia pelos produtores rurais.

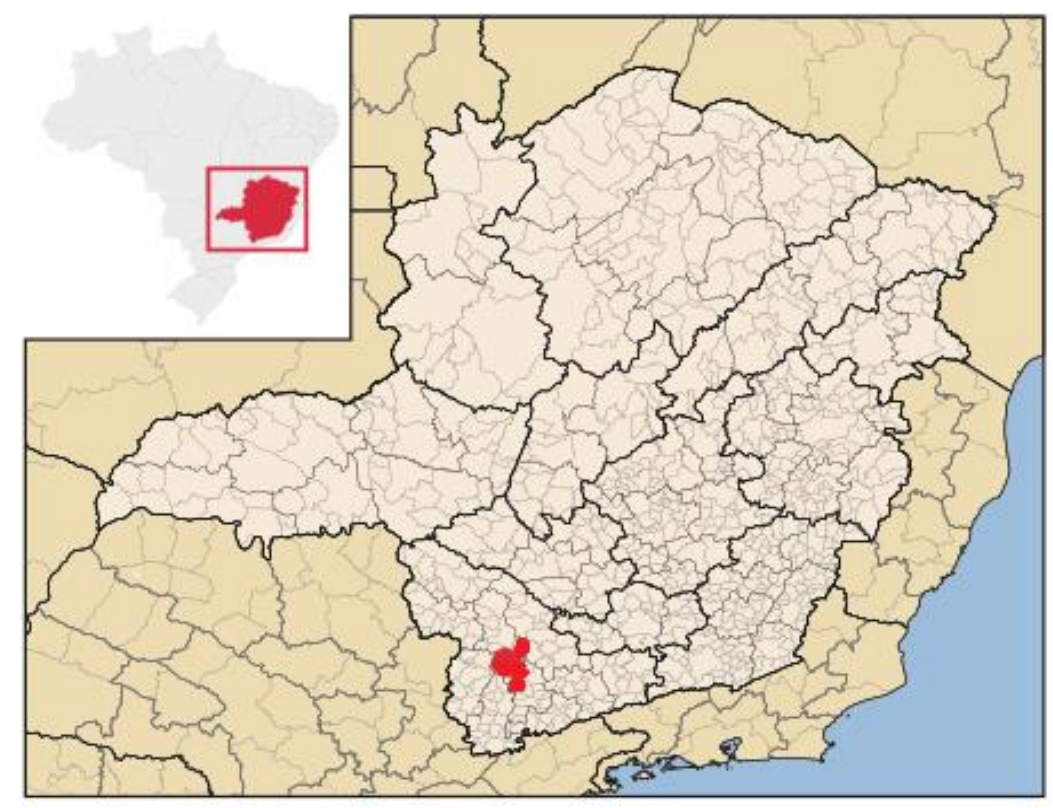

Figura 1: Mapa de Minas Gerais, região Sul, cidades estudadas.

Empregou-se métodos quantitativos para coleta e análise dos dados, uma vez que, o uso desse método possibilita ao pesquisador enfatizar a natureza social da construção da realidade, colocando em relevo o modo pelo qual a experiência social surge e adquire significado aos envolvidos (DENZIN; LINCOLN, 1994).

A pesquisa de campo baseou-se em pesquisas bibliográficas a fim de identificar temas relevantes para elaboração do questionário. Após este procedimento, construiu-se um região sul do estado de Minas Gerais, das cidades de Carvalhópolis, Machado, Paraguaçu, Poço Fundo, Silvianópolis e Turvolândia (Figura 1). 
Questão 2: Escolaridade: ( )Fundamental ( ) Médio; ( ) Ensino Superior ; ( )PósGraduação.

Questão 3: Há quanto tempo é produtor? Questão 4: Reside na propriedade rural?

( ) $\operatorname{sim}$ ( ) não
Questão 5: Se não, qual a distância entre moradia e propriedade

( ) alta ( ) média ( ) pequena

Questão 6: Onde é a propriedade?

Questão 7: Área destinada ao café:

Quadro 1. Responder sim ou não para as questões relacionada a produtos e serviços tecnológicos:

\section{PERGUNTA Sim Não}

1. A Tecnologia da Informação me auxiliaria em meus processos de produção.

2. Utilizo a Internet para consultar a previsão do tempo.

3. Possuo Software (sistema de computador) para administração (contabilidade, custos).

4. Possuo Software (sistema de computador) para gerenciar a produção.

5. Possuo celular do tipo smartphone.

6. Possuo computador ou notebook.

7. Tenho acesso à Internet

8. Tenho habilidade em utilizar a Internet.

9. Tenho habilidade em utilizar Smartphone.

10. Meu café é certificado.

Quadro 2. Principais barreiras para a adoção da Tecnologia da Informação (pode ser mais de uma):

\begin{tabular}{|l|l|l|}
\hline ( ) custo & $(\quad$ ) cultura & $(\quad)$ conflitos internos \\
\hline ( ) disponibilidade & $(\quad$ ) privacidade e segurança & $(\quad$ ) falta de necessidade \\
\hline
\end{tabular}

\section{RESULTOS E DICUSSÃO}

A partir das respostas obtidas por meio da aplicação dos questionários, constatou-se que a maior parte dos produtores rurais das regiões estudadas, $66 \%$, residem nas propriedades rurais. Ressaltando assim, a importância da inclusão digital em áreas não urbanas.
Outro fator importante que foi pesquisado diz respeito à faixa etária dos produtores rurais. Desta forma, foi possível constatar que a maioria, possui faixa etária entre 37 a 50 anos, totalizando $30 \%$ (Figura 2). 


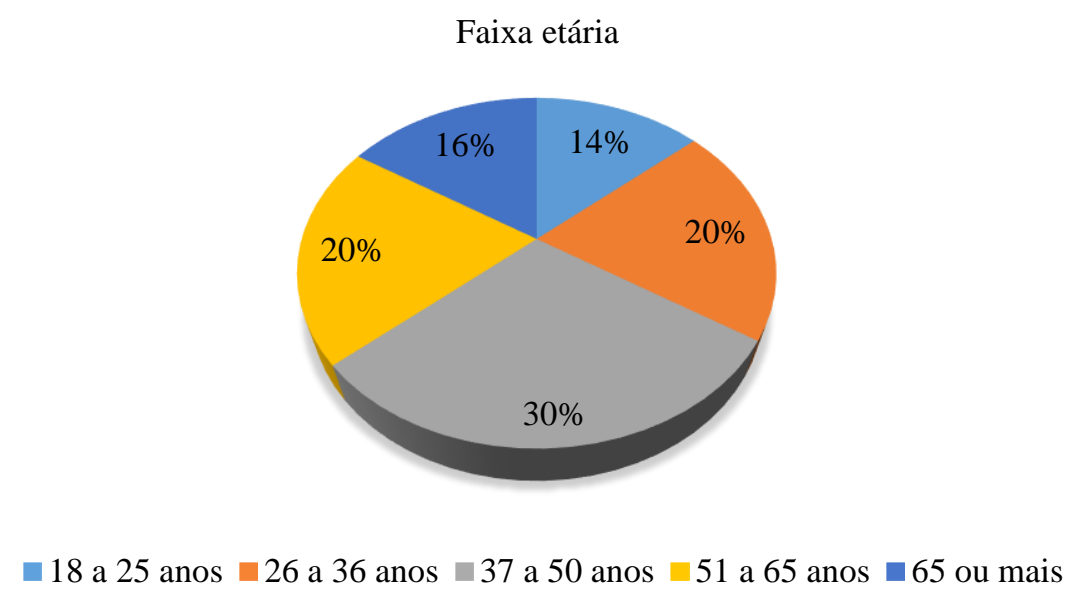

Figura 2: Faixa etária dos produtores rurais.

Para Bambini et al. (2015) um dos fatores que pode comprometer a adoção da Tecnologia da Informação por produtores rurais é o baixo nível de escolaridade. Quando se pergunta aos entrevistados, qual o nível de escolaridade, os produtores rurais, em sua maioria, têm apenas o Ensino Fundamental, correspondendo aproximadamente $57 \%$ dos entrevistados (Figura 3). Os produtores com formação superior, corresponde apenas $25 \%$ dos entrevistados.

Escolaridade

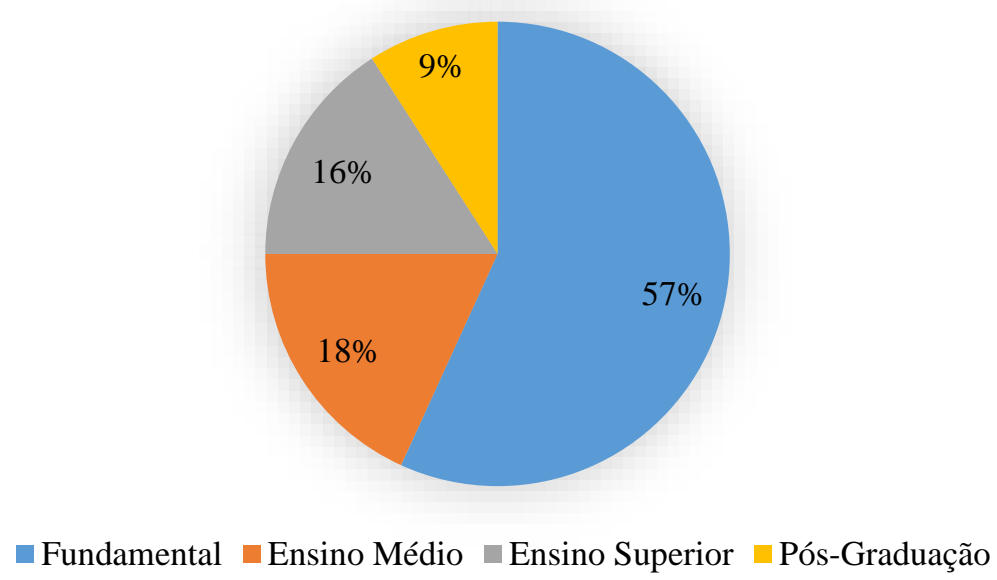

Figura 3: Nível de escolaridade dos produtores rurais.

A fim de detectar o acesso dos produtores às ferramentas tecnológicas, perguntou-se se possuem dispositivos como computador ou notebook e também se possuem celulares do tipo Smartphone. Os produtores que possuem computadores ou notebooks somam 59\% dos entrevistados. Em contrapartida, os produtores que possuem celulares do modelo Smartphones somam apenas $48 \%$ dos entrevistados.

$\mathrm{O}$ acesso à internet, há alguns anos, já era vista como algo indispensável em áreas urbanas, porém ela também se tornou uma ferramenta importante para o meio rural, tendo como objetivo fornecer 
conhecimento e informações atuais (VIERO; DA SILVEIRA, 2011). Por meio das pesquisas realizadas, pode-se observar que o acesso à Internet no meio rural, na região estudada, é amplo. Um total de $80 \%$ dos produtores entrevistados possui acesso à Internet. Este quesito é tão importante quanto terem acesso a um computador. Diante deste cenário, tornase possível os produtores adotarem sistemas informatizados para os auxiliarem em suas produções. Moreira et al. (2018) afirmam que as Tecnologias de Informação, como Internet, são avaliadas como alternativas significativas para possibilitar o compartilhamento de dados e informações.

Perguntou-se aos produtores se a Tecnologia da Informação poderia auxiliá-los em seus processos de produção, como, por exemplo, gestão do que foi produzido, controle financeiro, gestão de estoque e controle da produção. Nesta etapa a grande maioria acredita que a Tecnologia da Informação contribuiria em seus trabalhos, totalizando aproximadamente $73 \%$ (Figura 4).

A Tecnologia da Informação me auxiliaria em meus processos de produção.

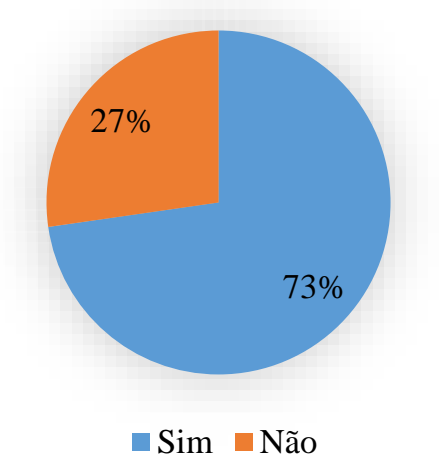

Figura 4: A Tecnologia da Informação auxiliaria os produtores rurais.

Em contrapartida, quando se questiona se utilizam algum software a maioria dos produtores rurais, cerca de $95 \%$, responde que não utiliza, ou seja, os produtores veem a Tecnologia da Informação como uma ferramenta que poderia auxiliá-los em suas produções, no entanto não fazem a utilização, conforme representa a Figura 5. Uma vez que, a grande maioria, possui computadores ou notebooks, estes produtores poderiam utilizar estes dispositivos já existentes para auxiliá-los, ou seja, podemos concluir que não é a falta de um computador que os impedem de terem softwares de gestão. 
Possuo Software para administração (contabilidade, custos)

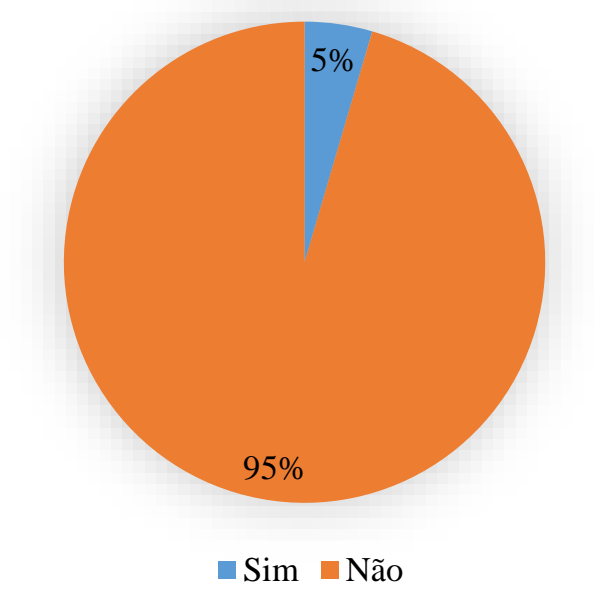

Figura 5: Utilização de software para controle da produção.

Para Viero; Da Silveira (2011), mesmo a Internet sendo uma ferramenta importante e influente, não basta apenas ter acesso a ela. É necessário saber utilizá-la em favor dos interesses e necessidades para implicar em mudanças nos indivíduos ou em comunidades. Desta forma, por meio das pesquisas realizadas, pode-se perceber que, os produtores entrevistados têm acesso à Internet, porém não têm explorado a utilização dela para atingir os objetivos e metas de suas produções rurais, buscando sistemas que os auxiliem em seus processos. A aplicação de sistemas informatizados no ambiente agrícola, tem expandido nos últimos anos, devido à ampliação de aquisições de dispositivos portáteis, como smartphones e tablets (CUNHA, 2017).

A fim de detectar o motivo de os produtores não adotarem a tecnologia, perguntou-se quais as principais barreiras para a adoção da Tecnologia da Informação. Foi possível constatar que a maioria dos produtores não possuem Tecnologia da Informação devido à falta de disponibilidade, cultura e alto custo para adquirir tal tecnologia. As opções somam mais de $100 \%$, pois o produtor poderia marcar mais de uma barreira (Figura 6). 
Barreiras para adoção da Tecnologia da Infomação

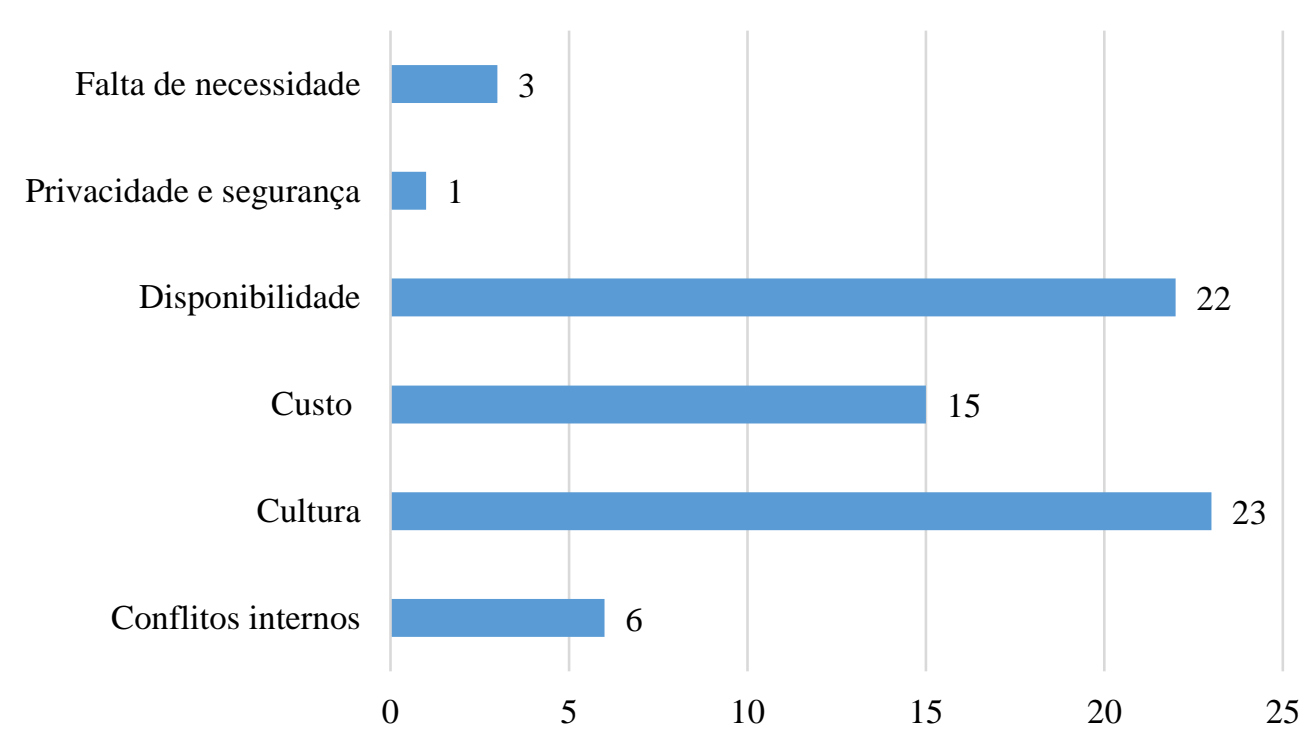

Figura 6: barreiras para a adoção da Tecnologia da Informação.

Entretanto, quando se diz respeito a consultas básicas na Internet, como por exemplo, previsão do tempo, grande parte dos entrevistados aderem a este procedimento. Totalizando $84 \%$ dos produtores que utilizam a Internet para consultar as condições climáticas.

Outro fator de suma importância questionado aos produtores diz respeito a habilidade em utilizar a Internet e Smartphones. Felizmente, $80 \%$ dos produtores dizem ter habilidade em utilizar Internet, ou seja, são familiarizados. Tão importante quanto ter conhecimento em utilizar a Internet é saber utilizar um Smartphone, uma vez que estes estão cada vez mais avançados e capazes de entregar aos produtores aplicativos para auxiliá-los. Um total de $75 \%$ dos produtores disse que sabe manusear um Smartphone. Este cenário auxilia bastante em futuras adoções em sistemas de gestão.

Segundo Bambini et al. (2015), mesmo as Tecnologias da Informação, trazendo notáveis contribuições, existem alguns fatores que limitam sua inserção no meio rural, como, por exemplo, falta de recursos financeiros, infraestrutura insuficiente em áreas com baixa densidade demográfica, carência de informações e competências para utilização e baixo nível escolar de pequenos agricultores e da agricultura familiar. Desta forma, existem programas governamentais que buscam levar o acesso à tecnologia para facilitar as operações ligada a gestão.

No mercado são disponibilizados aos agricultores, diversos tipos de softwares para as mais diversas finalidades. Cunha (2017), desenvolveu um sistema web com o objetivo de auxiliar os produtores de café na gestão de suas produções. Gabriel Filho et al. (2016) programaram um sistema informatizado capaz de, a partir da massa e altura de bovinos, identificar o estágio para abate. BADENKO; TERLEEV; VITALY (2014), exibiram em seu artigo um software de apoio à tomada de decisões na agricultura, sendo este capaz de estimar a situação agro meteorológica da cultura, prevendo assim a capacidade de rendimento das culturas, bem como traz contribuições para a tomada de decisões agrícolas. 


\section{CONCLUSÃO}

Diante do atual cenário competitivo no setor agropecuário, os produtores agrícolas deparam-se diante da necessidade de aderir inovações para sobressair e ter sucesso em seus processos de produções. Em contrapartida, diante dos estudos realizados no presente artigo, os pequenos e médios produtores, em sua maioria, ainda não possuem uma tecnologia computacional, como, por exemplo, softwares para gestão.

Pode-se perceber que os pequenos e médios agricultores, em sua maioria, utilizam a Internet para consultar

\section{AGRADECIMENTOS}

À Universidade José do Rosário Vellano pelo suporte acadêmico, a Faculdade de Ciências e Engenharias (UNESP/Tupã) e a Fundação de Amparo à Pesquisa de Minas Gerais (FAPEMIG).

\section{REFERENCIAS}

BADENKO, VLADIMIR; TERLEEV, VITALY; TOPAJ, Alexander. AGROTOOL software as an intellectual core of decision support systems in computer aided agriculture. Applied Mechanics \& Materials, 2014.

BAMBINI, M. D. et al. Software para agropecuária: panorama do mercado brasileiro. Parcerias Estratégicas, Brasília, v. 18, n. 36, p. 175-198, jan./jun. 2013.

CONFEDERAÇÃO DA

AGRICULTURA E PECUÁRIA DO

BRASIL. Agronegócio contribui para queda da inflação e geração de empregos em 2019. CNA/SENAR. 2019. Disponível em:

$<$ https://www.cnabrasil.org.br/boletins/pi previsões do tempo, mas não utilizam para fins de gestão. Grande parte dos entrevistados possuem computadores e acesso à Internet. Outro fator importante diz respeito às habilidades em utilizar a Internet e Smartphone, pode-se observar que a maioria dos produtores se caracterizaram com estas habilidades.

Diante destes fatores, torna-se mais simples a aquisição de um sistema para auxílio na gestão dos processos da produção agrícola. O que falta é a adaptação da cultura da propriedade e a procura por sistemas com custos mais baixos.

Agradecemos ao Conselho Nacional de Pesquisa (CNPq), bela concessão da Bolsa de Produtividade (Proc. 303923/2018-0) do último autor.

b-do-agronegocio-cresce-3-81-em-2019>. Acesso em 28 mar. 2020.

CUNHA, Angélica Carvalho et al. Computer system web for coffee production management control. Coffee Science, [S.1.], v. 12, n. 3, p. 344 - 354, set. 2017. ISSN 1984-3909. Disponível em:

$<$ http://www.coffeescience.ufla.br/index. php/Coffeescience/article/view/1291>. Acesso em: 16 nov. 2018.

DENZIN, Norman; LINCOLN, Yvonna. "Introduction: entering the field of qualitative research". In: Handbook of qualitative research. Londres: Sage, 1994.

GABRIEL FILHO, L. R. A. et al.

Software to assess beef cattle body mass through the fuzzy body mass index. 
Engenharia Agrícola, Jaboticabal, v. 36, p. 179-193, 2016.

GARCIA, J., VIEIRA FILHO, J.. Política agrícola brasileira: produtividade, inclusão e sustentabilidade. Revista de Política Agrícola, Local de publicação (editar no plugin de tradução o arquivo da citação ABNT), 23, abr. 2014. Disponível em:

$<$ https://seer.sede.embrapa.br/index.php/ RPA/article/view/888/813>. Acesso em: 16 Nov. 2018.

MOREIRA, Fábio Mosso et al. Construção Colaborativa de Representações para a Disseminação de Dados Agrícolas: Um Estudo do Portal CoDAF. Encontros Bibli: revista eletrônica de biblioteconomia e ciência da informação, v. 23, n. 52, p. 61-72, 2018.

VIERO, Verônica Crestani; DA SILVEIRA, Ada Cristina Machado. Apropriação de tecnologias de informação e comunicação no meio rural brasileiro. Cadernos de Ciência \&

Tecnologia, v. 28, n. 1, p. 257-277, 2011. Disponível em:

$<$ https://seer.sct.embrapa.br/index.php/cct /article/view/12042>. Acesso em 03 ago. 2018. 